\title{
COMPORTAMIENTO EN TERRENO DE PLANTAS DE QUILLAY (Quillaja saponaria Mol.), PRODUCIDAS EN DIFERENTES VOLÚMENES DE CONTENEDOR
}

\author{
Quiroz, I. ${ }^{1}$, Hernández, A. ${ }^{1}$, García, E. ${ }^{1}$, Gonzalez ${ }^{1}$, M. y Soto, H. $^{1}$
}

\section{RESUMEN}

Quillay (Quillaja saponaria Mol.) es una especie endémica de Chile, que se distribuye preferentemente en la zona mesomórfica del país, donde se encuentra sujeta a fuertes intervenciones para la obtención de productos no maderables destinados a los mercados farmacéutico y cosmetológico.

La presión sobre el recurso ha contribuido a que estas formaciones naturales estén en un estado de degradación y sobreexplotación que hace necesario recuperarlas favoreciendo su regeneración natural y estableciendo plantaciones con esta especie, tratamientos silviculturales que son objeto de incentivos bajo la legislación de fomento forestal vigente.

En plantaciones forestales, el rápido crecimiento de las plantas en terreno durante los primeros años es fundamental para su supervivencia, en especial en sitios edafoclimáticamente desfavorables, y en esto el sistema radicular formado en vivero cumple un papel preponderante, mejorando el prendimiento y el crecimiento inicial de las plantas por una mayor capacidad de arraigamiento y absorción de humedad desde el suelo.

El presente trabajo entrega resultados de investigaciones realizadas por INFOR en el área de plantaciones, probando contenedores de diferentes volúmenes para la producción de plantas en vivero y su efecto sobre el prendimiento y desarrollo inicial de estas en terreno.

Los volúmenes de los contenedores afectaron el crecimiento en diámetro $(\mathrm{P}<$ $0.05)$ y la altura $(P<0.05)$ de las plantas en terreno, aumentando ambas variables a medida que se incrementan los volúmenes de los contenedores, a un año de establecida la plantación.

Palabras clave: Volumen de contenedor, Quillaja saponaria, producción de plantas, plantación. 


\section{SUMMARY}

Quillay (Quillaja saponaria Mol.) is a native and endemic species in Chile with a natural geographic distribution mainly in the semiarid central part of the country where is currently under an intensive use to obtain non wood products for pharmaceutical and cosmetics markets.

Due to that pressure over the species, the Quillay forest are overexploited and degraded at present and it is necessary to recover them by favoring natural regeneration and through afforestation, both silvicultural treatments considered on the current forest law for State incentives.

The fast growth of seedlings during the first years in the establishment of forests plantations is essential for an appropriate survival of the stand, particularly under unfavorable soil or climate sites, where seedlings with a well developed root system will perform better, due to a good rooting and water utilization capacity.

This paper presents results from a research developed by INFOR on afforestation with Quillay using seedlings produced in different volume containers and assessing the seedling quality produced under this variable and later their survival and initial growth in the field a year after the establishment of the plantation.

The container volume shows an effect over the diameter growth $(P<0.05)$ and the total height $(P<0.05)$ of the seedlings one year after the plantation, and the effect is higher as the container volume is higher.

Key words: Container volume, Quillaja saponaria Mol., seedling production, plantation. 


\section{INTRODUCCIÓN}

Quillaja saponaria Mol. es una especie endémica de Chile que se ubica en la zona mesomórfica de del país y está adaptada para vivir en sitios pobres, secos y cálidos (Donoso, 1990). Crece desde los $100 \mathrm{msnm}$ en la Cordillera de la Costa hasta los 1.500 msnm en la Cordillera de los Andes.

Pese a no tener grandes restricciones climáticas para su desarrollo, existen actualmente problemas en su estado de conservación debido a las fuertes intervenciones a que está sometida para la obtención de productos no maderables destinados a los mercados farmacéutico y cosmetológico, lo que ha conducido a que las formaciones naturales de esta especie se encuentren en general en estado de degradación (Santelíces y Bobadilla, 1997).

Además del uso adecuado del recurso, bajo planes de manejo que aseguren su sostenibilidad, las técnicas silvícolas aplicables para la recuperación de las formaciones naturales de la especie son principalmente favorecer la regeneración natural de los bosques y la plantación, sea esta última orientada al enriquecimiento de los bosques existentes o a la creación de nuevos bosques mediante la forestación de áreas ya desarboladas.

En el establecimiento de plantaciones, una buena calidad de plantas es fundamental para la obtención de una adecuada supervivencia de estas en el terreno y un buen desarrollo inicial. La calidad de las plantas se refleja en su capacidad de arraigamiento y de aprovechamiento del agua disponible en el suelo, características particularmente gravitantes en sitios con limitaciones de suelo y clima como los habituales en las zonas semiáridas donde se desarrolla la especie.

Se ha demostrado que plantas de mayor tamaño tienen un mejor comportamiento en terreno (South, 1993; Dey y Parker ,1997), particularmente en condiciones de competencia con las malezas por recursos hídricos (South y Mitchell, 1999), existiendo una alta correlación entre el volumen del contenedor y la altura de la planta.

Según Close et al. (2010), plantas producidas en vivero en contenedores de mayor volumen podrían tener ventajas como un menor daño a las plantas durante el transporte y la plantación, una alta relación raíz: tallo, si el crecimiento superior es igual entre las plantas producidas en los diferentes contenedores; y una alta biomasa total con una similar relación raíz: tallo entre las plantas producidas en los distintos volúmenes de contenedores. A nivel fisiológico, la alta relación raíz: tallo puede dar lugar a relaciones más favorables en la absorción del agua, es decir menor necesidad en sus requerimientos de absorción, y tasas de crecimiento mayores (Close et al., 2010). Por otro lado, mayor biomasa total puede resultar en mayores reservas de carbohidratos totales disponibles para la re-movilización para el rápido crecimiento poco después de la plantación.

Actualmente, la decisión del tipo de contendor a utilizar depende de factores de manejo en vivero y de mercado, más que del análisis particular de su influencia en determinadas dimensiones; sin embargo, cuando el volumen del contenedor y la densidad 
de cultivo son los factores fundamentales de calidad de la planta en terreno, es conveniente conocer las tendencias de los factores dimensionales de cada planta según las variables de los contenedores (Peñuelas y Ocaña, 1999).

En virtud de la discrepancia existente en el beneficio obtenido en la calidad de planta y en el beneficio económico, considerando los escasos antecedentes técnicos que se disponen sobre producción de plantas nativas y más aún sobre su posterior desarrollo en terreno, surge la necesidad de determinar el efecto del volumen del contenedor en el crecimiento de las plantas en vivero y su comportamiento en terreno, motivo de este trabajo.

\section{MATERIAL Y MÉTODO}

El estudio fue realizado en el vivero del Centro Tecnológico de la Planta Forestal, perteneciente al Instituto Forestal ( $36^{\circ} 50^{\prime} 61^{\prime \prime}$ LS; $73^{\circ} 07^{\prime}$ 56" LO, $18 \mathrm{msnm}$ ). Las plantas de $Q$. saponaria fueron producidas desde semillas procedentes de la Región del Maule,

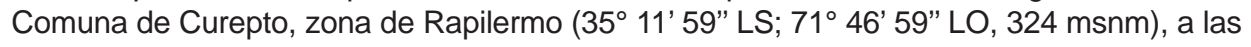
cuales se les aplicó un tratamiento pre-germinativo consistente en el remojo en agua por $24 \mathrm{~h}$.

En promedio, el peso medio de 100 semillas fue de $0,964 \mathrm{~g}$ y el número de semillas por kilogramo de 103.630 semillas (ISTA, 1996). La germinación fue de 95,2 $\pm 0,98 \%$, la energía germinativa de $85,4 \% \pm 3,5$, el vigor germinativo de $6,9 \% \pm 0,7$ días y el periodo de energía fue de 30,8 \pm 2,2 días (González et al., 2011).

La siembra se efectuó el 4 de junio 2009 en un sustrato de corteza de pino compostada de granulometría $\mathrm{G}$ - 10, en bandejas de polietileno expandido (Aislapol) en diferentes volúmenes de contenedores (Cuadro $N^{\circ} 1$ ), dispuestas en invernadero de polietileno UV nacional niquelado de $200 \mathrm{mc}$ durante dos meses, para luego ser trasladadas a un ambiente a cielo abierto.

\section{Cuadro $\mathrm{N}^{\circ} 1$ \\ CARACTERÍSTICAS DE LOS CONTENEDORES UTILIZADOS EN EL ESTUDIO}

\begin{tabular}{|c|c|c|c|c|c|}
\hline $\mathbf{N}^{\circ}$ & $\begin{array}{c}\text { Distribución de } \\
\text { Cavidades } \\
\text { Almaciguera }\end{array}$ & $\begin{array}{c}\text { Cavidades } \\
\text { Almaciguera } \\
\left(\mathbf{N}^{\circ}\right)\end{array}$ & $\begin{array}{c}\text { Cavidades } \\
\text { por Unidad de } \\
\text { Superficie } \\
(\mathbf{N} / \mathbf{m} \mathbf{2})\end{array}$ & $\begin{array}{c}\text { Volumen } \\
(\mathbf{c m} 3)\end{array}$ & $\begin{array}{c}\text { Profundidad } \\
(\mathbf{m m})\end{array}$ \\
\hline 1 & $13 \times 8$ & 104 & 416 & 56 & 70 \\
\hline 2 & $15 \times 9$ & 135 & 540 & 75 & 115 \\
\hline 3 & $14 \times 8$ & 112 & 448 & 95 & 140 \\
\hline 4 & $12 \times 7$ & 84 & 336 & 100 & 100 \\
\hline 5 & $12 \times 7$ & 84 & 336 & 130 & 160 \\
\hline 6 & $12 \times 7$ & 84 & 336 & 135 & 100 \\
\hline 7 & $11 \times 8$ & 88 & 400 & 140 & 135 \\
\hline 8 & $10 \times 6$ & 60 & 240 & 280 & 140 \\
\hline
\end{tabular}

(Fuente: González et al. 2011). 
El programa de riego consideró la aplicación de tres riegos diarios de un minuto, manteniendo el sustrato a una humedad del $80 \%$. El riego es tecnificado y se efectúa en forma automática mediante aspersión por "microjet". Las aplicaciones y tipo de fertilizantes se realizaron de acuerdo al estado de desarrollo de las plantas, según se indica en el Cuadro $\mathrm{N}^{\circ} 2$.

Cada 30 días, durante nueve meses (9 de septiembre del 2009 hasta 9 de mayo del 2010), fueron realizadas mediciones de crecimiento en altura $( \pm 0,1 \mathrm{~cm})$. Al final de la temporada de crecimiento ( 9 de mayo), cinco plantas de cada uno de los tratamientos y repetición fueron colectadas para la determinación del diámetro $( \pm 0,1 \mathrm{~mm})$ y secadas a $105^{\circ} \mathrm{C}$ hasta llegar a peso constante, para la determinación de biomasa aérea $( \pm 0,1 \mathrm{~g})$ y radicular $( \pm 0,1 \mathrm{~g})$.

\section{Cuadro $\mathrm{N}^{\circ} 2$ \\ APLICACIÓN DE FERTILIZANTE EN VIVERO}

\begin{tabular}{|l|c|l|}
\hline Etapa de Crecimiento & $\begin{array}{c}\text { Elementos } \\
\text { N-P-K }\end{array}$ & \multicolumn{1}{c|}{ Aplicación } \\
\hline Hasta $10 \mathrm{~cm}$ & $15 \% ; 30 \% ; 15 \%$ & Una vez por semana en dosis de 2 gramos/litro \\
\hline Desde 10 - Hasta $25 \mathrm{~cm}$ & $25 \% ; 10 \% ; 10 \%$ & Una vez por semana en dosis de 3 gramos/litro \\
\hline Sobre $25 \mathrm{~cm}$ & $\begin{array}{c}13 \% ; 6 \% ; 40 \% \\
\text { (mas nitrato de calcio) }\end{array}$ & $\begin{array}{l}\text { Una vez por semana en dosis de } 2 \text { gramos/litro. Nitrato } \\
\text { de Calcio dos gramos/litros dos veces por semana. }\end{array}$ \\
\hline
\end{tabular}

Para la instalación del ensayo de evaluación del comportamiento de las plantas en terreno (1 de agosto del 2010), se realizó una limpieza del sector mediante un roce de las malezas, las cuales fueron ordenadas en fajas. La plantación se realizó en casillas mediante la remoción de suelo de $40 \times 40 \times 40 \mathrm{~cm}$ y a una densidad de 1.100 plantas /ha (3 x $3 \mathrm{~m}$ ). Un total de 972 plantas fueron establecidas en la Región del Bíobío, Comuna de Florida, Chile coordenadas (36 47' 54" LS; 72 39' 61" LO, 238 msnm). Finalmente la primera semana de Septiembre 2010, se fertilizó con 140 gramos de una mezcla forestal (N 8\% P $20 \%$ K 7\%).

El diseño experimental correspondió a bloques completos al azar con tres repeticiones, considerando ocho volúmenes de contenedores $(56,75,95,100,130,135$, 140 y $280 \mathrm{~cm}^{3}$ ), donde la unidad muestral estuvo compuesta por 36 plantas. Se realizaron mediciones de crecimiento en diámetro y altura al inicio del establecimiento del ensayo y al primer año (1 de junio del 2011).

Al final del período de crecimiento en terreno fueron realizados análisis de varianza (ANDEVA) para evaluar diferencias en longitud de tallo (LT), diámetro de cuello de la raíz (DAC). La homogeneidad de varianza fue evaluada mediante la prueba de Levene $(P<$ 0,05).

El supuesto de normalidad de los residuos se evaluó mediante la prueba de Shapiro- 
Wilks $(P<0,05)$. Para detectar diferencias significativas entre los tratamientos, se realizó la prueba de comparación múltiple Scott \& Knott, con un $95 \%$ de confianza.

Se evalúo la correlación existente entre los parámetros morfológicos con el volumen y altura del contenedor. Un análisis de correlación cruzado fue realizado para analizar el grado de asociación entre las variables que fueron evaluadas, mediante los valores de significancia a un nivel de probabilidad $(P)$ y coeficientes de correlación de Pearson $\left(\mathrm{r}^{2}\right)$.

Se identificaron en cada caso las variables independientes significativamente correlacionadas con la variable de respuesta $\mathrm{P}<0,05$. Los datos fueron analizados utilizando el software estadístico InfoStat (2008).

\section{RESULTADOS Y DISCUSIÓN}

Al final de la etapa de vivero, las plantas mostraron diferencias significativas entre los tratamientos $(P<0.05)$. Las plantas que crecieron con un mayor volumen por cavidad mostraron diferencias significativas $(P<0.05)$ en altura, diámetro, longitud de la raíz principal, biomasa e índices de calidad, comparadas con aquellas producidas en contenedor de 56 $\mathrm{cm}^{3}$ (Cuadro $\mathrm{N}^{\circ} 2$ ).

Plantas producidas en contenedores de $280 \mathrm{~cm}^{3}$ y $135 \mathrm{~cm}^{3}$ presentaron valores similares en altura de 26,4 y $26,2 \mathrm{~cm}$, respectivamente, a pesar de la diferencia existente en volumen y altura del contenedor (González et al., 2011).

La Norma Chilena NCh 2957/5 de calidad de planta, no considera estándares de calidad para la especie $Q$. saponaria, sin embargo al realizar una comparación con los estándares normativos de raulí producida a raíz cubierta, sólo el contendor de $56 \mathrm{~cm}^{3}$ no cumpliría con la normativa (INN, 2006).

Para las variables de biomasa, existe una tendencia en directa relación con el volumen del contenedor (Cuadro $\mathrm{N}^{\circ} 3$ ). A mayor volumen de contenedor mayor peso seco aéreo y radicular. Sólo el contenedor de $140 \mathrm{~cm}^{3}$ presenta un comportamiento diferente, presentando valores totales menores que los obtenidos en plantas producidas en contenedor de $135 \mathrm{~cm}^{3}$. 


\section{Cuadro $\mathrm{N}^{\circ} 3$ \\ EFECTO DEL VOLUMEN DEL CONTENEDOR EN LOS ATRIBUTOS MORFOLÓGICOS, BIOMASA E ÍNDICES DE CALIDAD DE PLANTAS} (media \pm error estándar; $n=15$ )

\begin{tabular}{|c|c|c|c|c|c|c|c|c|c|}
\hline \multirow{2}{*}{$\begin{array}{c}V \\
(\mathrm{~cm} 3)\end{array}$} & \multirow{2}{*}{$\begin{array}{c}\text { LT } \\
(\mathrm{cm})\end{array}$} & \multirow{2}{*}{$\begin{array}{l}\text { DAC } \\
(\mathrm{mm})\end{array}$} & \multirow{2}{*}{$\begin{array}{l}\text { L. raíz } \\
\text { (cm) }\end{array}$} & \multicolumn{3}{|c|}{$\begin{array}{c}\text { Biomasa } \\
\text { (g) }\end{array}$} & \multicolumn{3}{|c|}{ Índice de Calidad } \\
\hline & & & & Aérea & Raíz & Total & Esbeltez & $\begin{array}{l}\text { Tallo/ } \\
\text { raíz }\end{array}$ & Dickson \\
\hline 280 & $26,4 \pm 1,6 a$ & $4,8 \pm 0,2 a$ & $12,0 \pm 0,6 a$ & $3,5 \pm 0,3 a$ & $1,8 \pm 0,1 \mathrm{a}$ & $5,3 \pm 0,4 a$ & $4,9 \pm 0,3 a$ & $2,0 \pm 0,1 b$ & $0,8 \pm 0,0 a$ \\
\hline 140 & $20,9 \pm 1,4 b$ & $3,5 \pm 0,2 \mathrm{c}$ & $9,2 \pm 0,5 a$ & $2,2 \pm 0,2 b$ & $1,3 \pm 0,1 \mathrm{a}$ & $3,6 \pm 0,3 b$ & $6,0 \pm 0,2 b$ & $1,9 \pm 0,2 b$ & $0,5 \pm 0,0 a$ \\
\hline 135 & $26,2 \pm 1,5 a$ & $4,2 \pm 0,3 a$ & $6,8 \pm 0,9 c$ & $3,2 \pm 0,3 a$ & $1,4 \pm 0,2 a$ & $4,6 \pm 0,4 a$ & $6,7 \pm 0,4 a$ & $2,4 \pm 0,1 \mathrm{a}$ & $0,5 \pm 0,1 \mathrm{a}$ \\
\hline 130 & $22,2 \pm 1,4 \mathrm{~b}$ & $4,2 \pm 0,2 a$ & $9,4 \pm 1,1 \mathrm{a}$ & $2,2 \pm 0,2 b$ & $1,4 \pm 0,2 \mathrm{a}$ & $3,6 \pm 0,4 b$ & $5,5 \pm 0,4 a$ & $1,8 \pm 0,2 b$ & $0,5 \pm 0,0 a$ \\
\hline 100 & $21,3 \pm 0,7 b$ & $3,4 \pm 0,1 \mathrm{c}$ & $8,0 \pm 0,2 b$ & $2,0 \pm 0,1 b$ & $0,9 \pm 0,0 \mathrm{~b}$ & $2,9 \pm 0,1 \mathrm{c}$ & $6,4 \pm 0,3 a$ & $2,2 \pm 0,2 a$ & $0,4 \pm 0,0 b$ \\
\hline 95 & $19,2 \pm 1,4 \mathrm{c}$ & $3,7 \pm 0,2 b$ & $9,7 \pm 1,0 \mathrm{a}$ & $1,9 \pm 0,2 b$ & $0,9 \pm 0,1 b$ & $2,9 \pm 0,3 c$ & $5,2 \pm 0,2 a$ & $2,0 \pm 0,1 b$ & $0,4 \pm 0,0 \mathrm{~b}$ \\
\hline 75 & $18,1 \pm 1,4 \mathrm{c}$ & $3,4 \pm 0,2 \mathrm{c}$ & $9,5 \pm 0,6 a$ & $1,8 \pm 0,2 b$ & $0,8 \pm 0,1 b$ & $2,6 \pm 0,3 c$ & $5,9 \pm 0,4 a$ & $2,5 \pm 0,1 \mathrm{a}$ & $0,3 \pm 0,0 b$ \\
\hline 56 & $15,8 \pm 0,7 d$ & $2,9 \pm 0,2 d$ & $5,4 \pm 0,5 c$ & $1,5 \pm 0,2 b$ & $0,5 \pm 0,1 b$ & $2,0 \pm 0,2 \mathrm{c}$ & $5,3 \pm 0,4 a$ & $3,1 \pm 0,2 a$ & $0,2 \pm 0,0 \mathrm{~b}$ \\
\hline
\end{tabular}

(Fuente: Modificado de González et al., 2011).

$\mathrm{V}=$ volumen del contenedor, $\mathrm{LT}=$ longitud del tallo, DAC = diámetro de cuello, L. raíz = longitud de raíz.

Valores promedios, con la misma letra no difieren significativamente entre sí, P > 0,05.

Asimismo, existe una alta correlación entre los parámetros morfológicos, es decir a medida que aumenta el volumen y profundidad del contenedor mayores son los valores de dichos parámetros, característica que se presenta con mayor notoriedad en la longitud de la raíz (Cuadro $N^{\circ} 4$ ). Por el contrario, estos parámetros se ven afectados negativamente a medida que aumenta el número de cavidades por almaciguera y por unidad de superficie, principalmente en la altura y el diámetro.

\section{Cuadro $\mathrm{N}^{\circ} 4$ \\ CORRELACIÓN DE PEARSON ENTRE LOS PARÁMETROS MORFOLÓGICOS, BIOMASA E ÍNDICE DE CALIDAD, Y EL VOLUMEN Y PROFUNDIDAD DE LOS CONTENEDORES \\ (Significativo en $P>0,05$ )}

\begin{tabular}{|c|c|c|c|c|c|c|c|c|c|}
\hline \multirow{2}{*}{$\begin{array}{l}\text { Características } \\
\text { contenedor }\end{array}$} & \multirow{2}{*}{$\begin{array}{l}\mathrm{LT} \\
\text { (cm) }\end{array}$} & \multirow{2}{*}{$\begin{array}{l}\text { DAC } \\
(\mathrm{mm})\end{array}$} & \multirow{2}{*}{$\begin{array}{l}\text { L. } \\
\text { Raíz } \\
\text { (cm) }\end{array}$} & \multicolumn{3}{|c|}{$\begin{array}{l}\text { Biomasa } \\
\text { (g) }\end{array}$} & \multicolumn{3}{|c|}{ Índice de Calidad } \\
\hline & & & & Aérea & Raíz & Total & Esbeltez & Tallo/raíz & Dickson \\
\hline Volumen & 0,90 & 0,91 & 0,80 & 0,93 & 0,95 & 0,95 & $-0,16$ & $-0,38$ & 0,98 \\
\hline Altura & 0,49 & 0,65 & 0,83 & 0,45 & 0,68 & 0,53 & $-0,27$ & $-0,88$ & 0,64 \\
\hline
\end{tabular}

$\mathrm{LT}=$ longitud del tallo, $\mathrm{DAC}=$ diámetro de cuello, $\mathrm{L}$. raíz = longitud de raíz .

Al final de la etapa en vivero, el crecimiento de las plantas mostró diferencias significativas en altura, diámetro y biomasa entre los diferentes volúmenes de contenedor. Ortega et al. (2006) señalan que plantas de Pinus radiata creciendo en volúmenes de 200, 260 y $270 \mathrm{~cm}^{3}$ no muestran diferencias en crecimiento en altura, existiendo solo diferencias en el número de raíces secundarias producidas en la parte media y baja del contenedor, 
respuesta que podría ser consecuencia de la aireación en la parte lateral que presentan los contenedores y que favorecería la poda de raíces laterales.

Anthony et al. (2006) reportan que el tipo de contenedor afectó significativamente $(\mathrm{P}<0,05)$ la altura del tallo y la simetría del sistema radicular en plantas de Pinus taeda producidas mediante estacas.

No obstante, las contradicciones existentes entre los autores se deben a la existencia de un efecto del contenedor, cerrados y fisurados, $y$ al volumen y altura de este, en el crecimiento de la parte aérea y radicular de las plantas.

El volumen del contenedor y la biomasa de las plantas presentan una alta correlación, siendo descrito de forma similar en plantas de Picea mariana (Lamhamedi et al., 1998), demostrando que un contenedor con mayor capacidad volumétrica favorece el desarrollo de las plantas, pero asimismo el tipo de contenedor determina la arquitectura de las raíces, siendo un factor que afecta la formación de raíces secundarias, terciarias y la longitud de la raíz principal (Ortega et al., 2006).

\section{Cuadro $\mathrm{N}^{\circ} 5$ \\ EFECTO DEL VOLUMEN DEL CONTENEDOR EN LOS ATRIBUTOS MORFOLÓGICOS, SUPERVIVENCIA Y DAÑO PROVOCADO POR LOS CONEJOS (media \pm desviación estándar; $n=108$ )}

\begin{tabular}{|c|c|c|c|c|}
\hline $\begin{array}{c}\mathbf{V} \\
(\mathbf{c m} 3)\end{array}$ & $\begin{array}{c}\mathbf{L T} \\
\mathbf{( c m})\end{array}$ & $\begin{array}{c}\text { DAC } \\
(\mathbf{m m})\end{array}$ & $\begin{array}{c}\text { Supervivencia } \\
(\%)\end{array}$ & $\begin{array}{c}\text { Daño Conejo } \\
(\%)\end{array}$ \\
\hline 280 & $33,3 \pm 10,2 \mathrm{a}$ & $5,8 \pm 1,3 \mathrm{ab}$ & $98 \mathrm{a}$ & 15,7 \\
\hline 140 & $27,0 \pm 8,3 \mathrm{~cd}$ & $5,1 \pm 1,4 \mathrm{~cd}$ & $96 \mathrm{a}$ & 33,3 \\
\hline 135 & $34,8 \pm 9,0 \mathrm{a}$ & $6,1 \pm 1,5 \mathrm{a}$ & $99 \mathrm{a}$ & 5,6 \\
\hline 130 & $32,4 \pm 10 \mathrm{ab}$ & $5,7 \pm 1,6 \mathrm{abc}$ & $95 \mathrm{a}$ & 19,4 \\
\hline 100 & $27,8 \pm 8,4 \mathrm{c}$ & $5,4 \pm 1,5 \mathrm{bcd}$ & $100 \mathrm{a}$ & 15,7 \\
\hline 95 & $29,4 \pm 7,2 \mathrm{bc}$ & $5,5 \pm 1,1 \mathrm{bcd}$ & $100 \mathrm{a}$ & 24,1 \\
\hline 75 & $26,6 \pm 8,7 \mathrm{c}$ & $5,1 \pm 1,1 \mathrm{~cd}$ & $100 \mathrm{a}$ & 17,6 \\
\hline 56 & $23,4 \pm 7,4 \mathrm{~d}$ & $4,9 \pm 1,6 \mathrm{~d}$ & $99 \mathrm{a}$ & 14,8 \\
\hline
\end{tabular}

$\mathrm{LT}=$ longitud del tallo, DAC = diámetro de cuello.

Los índices de esbeltez, tallo/raíz, y de Dickson (Diskson et al., 1960), de las plantas de $Q$. saponaria producidas en los diferentes volúmenes de contenedor alcanzaron valores entre $6,7-4,9 ; 3,1-1,8$; y 0,9-0,2, respectivamente, mostrando el equilibrio entre la superficie que transpira (aérea) y la que absorbe agua (radicular) (Santelíces et al., 2011), favoreciendo así un mayor desarrollo del sistema radical que la parte aérea con lo que favorecería la sobrevivencia en zonas secas (Villar - Salvador, 2003).

García (2007) considera que el valor de esbeltez no debe ser mayor a 6. Así mismo Hunt (1990) menciona que la esbeltez debe ser menor o igual a 8 para plantas que se encuentran en una condición de equilibrio entre la altura y diámetro. 
Sin embargo, existen antecedentes de las características morfológicas que deberían tener las plantas para diferentes condiciones de sitio y ambiente, por ello Cleary et al. (1978) determinaron que plantas de mayor tamaño presentan un mejor desempeño en la supervivencia y crecimiento en condiciones de competencia con malezas. Asimismo, en condiciones de sitios favorables, plantas de mayor altura crecen mejor que plantas pequeñas (Iverson, 1984; Ritchie, 1984), sin embargo, plantas de mayor tamaño no sobreviven tan bien como las de menor tamaño (Thompson, 1984). Según Hunt (1990), un valor inferior a 0,15 en el índice de Dickson en abeto y pino, puede significar problemas en el establecimiento de las plantas, encontrándose las plantas desequilibradas, por ello para esas especies se recomienda un índice de Dickson superior a 0,2.

Los antecedentes obtenidos en vivero para $Q$. saponaria muestran que los tamaños de los contenedores afectan las variables de altura de tallo, diámetro de cuello y pesos secos, tanto aéreo, de raíz y total, sin embargo dichas diferencias no influyeron en las supervivencias de las plantas en terreno. En tal sentido, parece conveniente, desde el punto de vista del viverista y forestador, utilizar plantas producidas en contenedores de 130 o 135 $\mathrm{cm}^{3}$, ello permitiría indicar que contenedores de mayor volumen no serían necesarios. No obstante, en un estudio de plantas de Eucalyptus grandis Hill ex Maiden agrupadas en cuatro clases morfológicas, basadas en altura y diámetro, no se registraron diferencias significativas en el crecimiento y supervivencia en campo, pero plantas con mayor altura y diámetro fueron más susceptibles a las bajas temperaturas (García, 2007).

Plantas producidas en contenedores de menor volumen, con sistemas radiculares poco desarrollados, conducen a un menor crecimiento, sin embargo contenedores con mayor volumen y altura del contenedor, mejoraron el crecimiento de las plantas en terreno, aumentando la absorción de humedad desde el suelo a través de las raíces, teniendo éstas acceso a humedad a mayor profundidad (Close et al., 2010). Se mejora así el crecimiento de nuevas raíces después del establecimiento en terreno, evitando el estrés hídrico, manteniendo las tasas fotosintéticas, y asegurando el éxito del establecimiento (Burdett, 1990) y cumpliendo variadas funciones en la fisiología de la planta, tales como la absorción de agua y nutrientes desde el suelo, el transporte de éstos a los tallos y hojas, y la síntesis de hormonas vegetales (Salisbury y Ross, 2000).

En el ensayo establecido con $Q$. saponaria se observó un aumento significativo en la altura y diámetro de las plantas $(P<0,05)$ transcurrido un año del establecimiento en terreno (Cuadro $\mathrm{N}^{\circ} 3$ ), sin embargo estas diferencias en el crecimiento se reducen durante el transcurso del tiempo, desde el momento que las plantas son recolectadas en vivero, sin embargo se obtiene un mayor varianza, especialmente en la altura, lo que puedes ser influenciado por las particularidades del sitio.

Un ensayo con Eucalyptus globulus Labill establecido en la comuna de Lumaco, transcurridos 4 años de establecido en terreno, mostró que las diferencias iniciales de crecimiento entre los diferentes contenedores no fueron significativas (datos no publicados), por lo que se debe esperar otras evaluaciones que permitan confirmar el comportamiento de $Q$. saponaria y el efecto de los contenedores. 
En términos de supervivencia, no se observaron diferencias significativas entre los tratamientos, ya que esta fue alta, sin embargo algunos tratamientos fueron afectados por conejos, no existiendo una relación entre la altura de la planta y el ataque. Las plantas cortadas presentaban rebrotes y fueron eliminadas del análisis.

En general y al igual que en otras investigaciones (Domínguez et al., 1997; Domínguez, 2000), se puede indicar que los contenedores de mayor volumen presentaron mejores resultados de crecimiento a un año de establecido en terreno. Se observa que los contenedores de mayor profundidad son los que presentaron una tendencia a mayor crecimiento en altura, en particular los contenedores de $130 \mathrm{~cm}^{3}$.

\section{CONCLUSIONES}

A un año de establecido el ensayo en terreno, se puede indicar que los volúmenes de los contenedores afectaron significativamente el crecimiento en diámetro y la altura de las plantas, aumentando ambas variables a medida que se incrementan los volúmenes de los contenedores.

Las diferencias iniciales de crecimiento obtenidas en vivero en las variables diámetro y altura, transcurridos un año en terreno tienden a disminuir, aunque se obtiene una mayor varianza en dichas variables.

No existe una relación entre el tamaño de la planta de $Q$. saponaria establecida en terreno y su sobrevivencia.

Evaluaciones posteriores permitirán apreciar si las diferencias se mantienen a más edad de la plantación.

\section{REFERENCIAS}

Anthony, V., Barry, G., Frank, B., Jeff, W., Ben, C., Farrell, W. and John, F., 2006. Container type volume influences adventitious rooting and subsequent field growth of stem cuttings of Loblolly Pine. Society of American foresters 30 (3): $123-131$.

Burdett, A., 1990. Physiological processes in plantation establishment and the development of specifications for forest planting stock. Canadian Journal of Forest Research 20:415427.

Cleary, B., Greaves, R., and Owston, W., 1978. Seedlings. P. 63-98 in: Regenerating Oregon's Forests, B. D. Cleary, R. D. Greaves, and R. K. Hermann (eds.). Oregon State University Extension Service, Corvallis. 287 p.

Close, D., Paterson, S., Corkrey, R., and Mc Arthur, C., 2010. Influence of seedling size, container type and mammal browsing on the establishment of Eucalyptus globulus in plantation forestry. New Forests 39:105-115. 
Dey, D. and Parker, W., 1997. Morphological indicators of stock quality and field performance of Red Oak (Quercus rubra L.) seedlings underplanted in a central Ontario shelterwood. New Forests 14:145-156

Dickson, A., Leaf, A. and Hosner, I., 1960. Quality appraisal of White Spruce and White Pine seedlings stock in nurseries. Forestry Chronicle 36: 10-13.

Dominguez S., Herrero, N., Carrasco, I., Ocaña, L. y Rubira J., 1997. Ensayos de diferentes tipos de contenedores para Quercus ilex, Pinus halapensis, Pinus pinaster y Pinus pinea: Resultado de vivero. Actas de II congreso Forestal Español Pamplona Mesa 3: 189-194.

Dominguez S., 2000. Influencia de distintos tipos de contenedores en el desarrollo en campo de Pinus halepensis y Quercus ilex. Reunión de Coordinación I+D. Fundación CEAM.

Donoso, C., 1990. Ecología Forestal, el bosque y su medio ambiente. Editorial Universitaria, Segunda edición, Santiago de Chile, 367p.

García, M., 2007. Importancia de la calidad del plantín forestal. XXII Jornadas Forestales de Entre Ríos. Argentina, Octubre de 2007. Disponible en: http://www.inta. gov.ar/concordia/info/Forestales/contenido/pdf/2007/312.II.GARCIA.pdf (con acceso el 02-08-2010).

González, M., Quiroz, I., García, E. y Soto, H., 2011. Estándares de producción de plantas de Quillay (Quillaja saponaria Mol.) Revista Chile Forestal 353: 43 - 46.

Iverson, R., 1984. Planting stock selection: Meeting biological needs and operational realities. In Duryea ML, TD Landis eds. Forest nursery manual. Oregon State University. Corvallis, USA. p. 261-266.

Hunt, G., 1990. Effect of styroblock design and copper on morphology of conifer seedlings. En: Rose, R., S. J. Campbell y T. D. Landis (eds.). Proceedings, Western Forest Nursery Association; 1990 August 13-17; Roseburg, OR. General Technical Report RM-200. Fort Collins, CO: U.S. Department of Agriculture, Forest Service, Rocky Mountain Forest and Range Experiment Station: 218-222. Disponible en: www.rngr.net/publications/ proceedings/ 1990/hunt.pdf/at_download/file (con acceso el 30 de julio de 2008).

INN, 2006. Norma Chilena Oficial. NCh 2957. Of2006. Material de propagación de uso forestal. 1ra ed. Instituto Nacional de Normalización, Santiago, Chile.

InfoStat, 2008. InfoStat versión 2008. Grupo InfoStat, Facultad de Ciencias Agrarias, Universidad Nacional de Córdoba. Córdoba, Argentina.

ISTA, 1996. International rules for seed testing. Seed Sci. Technol., 24: Supplement.

Lamhamedi, M., Bernier, P., Hebert, C. and Jobidon, R., 1998. Physiological and growth 
responses of three sizes of containerized of Picea mariana seedlings outplanted with and without vegetation control. Forest Ecology and Management 110: 13-23.

Ortega, U., Majada, J., Mena-Petite, A., Sanchez-Zabala, N., Rodriguez-Iturrizar, N., Txarterina, K., Azpitarte, J. and Duñabeitia, M., 2006. Field performance of Pinus radiata D. Don produced in nursery with different types of containers. New Forests 31: 97-112.

Peñuelas, J. y Ocaña, L., 1999. Cultivo de plantas forestales en Contenedor. Ministerio de Agricultura, Pesca y Alimentación. Ediciones Mundi-Prensa. 190 pág.

Ritchie, G., 1984. Assessing seed quality. In: Duriyea, M.L.: Landis, T.D., eds. Forest nursery manual: production of bareroot seedlings. Boston: Kluwer Academic Publishers: 243-259.

Salisbury, F. y Ross, C., 2000. Fisiología Vegetal. Madrid, España. Thomson Editores, Spain Paraninfo. 988 p.

Santelices, R., Navarro, R., Drake, F. y Mena, C., 2011. Efecto de la cobertura y de la fertilización en el desarrollo de plantas de Nothofagus alessandrii cultivadas en contenedor. Bosque 32 (1): $85-88$.

Santelices, R. y Bobadilla, C., 1997. Arraigamiento de estacas de Quillaja saponaria Mol. y Peumus boldus Mol. Bosque 18 (2): 77 - 85.

South, D., 1993. Rationale for growing Southern Pine seedlings at low seedbed densities. New Forests 7:63-92.

South, D. and Mitchell, R., 1999. Determining the "optimum" Slash Pine seedling size for use with four levels of vegetation management on a flatwoods site in Georgia. USA. Canadian Journal of Forest Research 29:1039-1046.

Thompson, B., 1984. Seedling morphological evaluation-what you can tell by looking. In: Duryea MI (Eds.), Evaluating Seedling Quality: Principles Procedures and Predictive Abilities of Major Tests. Oregon State University, USA, pp. 59-72.

Villar-Salvador, P., 2003. Importancia de la calidad de planta en los proyectos de revegetación. In Rey-Benayas JM, TEspigares-Pinilla, JM Nicolau-lbarra eds. Restauración de ecosistemas mediterráneos. Alcalá de Henares, España. Universidad de Alcalá. p. 6586. 